\title{
O modelo atômico de Bohr e as suas limitações na interpretação do espec- tro do átomo de hélio ${ }^{+*}$
}

\author{
Danielle Nicolodelli Tenfen ${ }^{1}$ \\ Wagner Tenfen ${ }^{2}$ \\ Universidade Federal da Fronteira Sul \\ Realeza - PR
}

\section{Resumo}

A inserção de tópicos de física moderna e contemporânea na Educação Básica já não é mais algo tão distante. Os livros didáticos aprovados no último Plano Nacional contemplam, de formas distintas, discussões a esse respeito. Porém, sabe-se que a efetiva implementação desses conteúdos em sala de aula depende da formação - tanto conceitual quanto pedagógica - dos professores. Nesse sentido, o presente artigo visa contribuir com professores e estudantes de graduação em Física, apresentando um breve delineamento histórico que enfatiza o contexto de surgimento do modelo atômico de Niels Bohr, conhecido por situar-se em um periodo de transição entre Física Clássica e Moderna. Ainda, mostram-se detalhadamente as limitações do modelo de Bohr, evidenciando a necessidade das explicações oriundas da nova Mecânica Quântica para uma descrição adequada do espectro do átomo de hélio. Por fim, apresentase uma proposta experimental que consiste na construção de um espectroscópio de baixo custo e problematizam-se alguns dados coletados a partir desse aparato.

Palavras-chave: Modelo atômico de Bohr; Átomo de hélio; Espectroscopia.

\footnotetext{
${ }^{+}$Bohr atomic model and its limitations on the interpretation of helium atomic spectrum

* Recebido: março de 2016. Aceito: outubro de 2016.

${ }^{1}$ E-mail: dntenfen@gmail.com

${ }^{2}$ E-mail: wtenfen@gmail.com
} 


\begin{abstract}
The insertion of modern and contemporary Physics topics on Brazilian High School Education is no longer something so distant. The didactic books approved in the last National Plan include, somehow, discussions about this theme. However, the effective implementation of these subjects in schools depends on conceptual and didactic training of the teachers. In this sense, the presente article intends to contribute with teachers and undergraduate Physics students by presenting a brief historical delimitation which emphasizes the context where Bohr atomic model emerges, known to be proposed in a period of transition between classical and modern Physics. Further, we show in details the limitations of this model, pointing the need of explanations from the new quantum mechanics to correctly describe the Helium atomic spectrum. Finally, we present an experimental proposal for the construction of a low cost spectroscope, and discuss some data collected with this equipment.
\end{abstract}

Keywords: Bohr atomic model; Helium atom; Spectroscopy.

\title{
I. Introdução
}

O Plano Nacional do Livro Didático (PNLD) de 2015, na área de Física, é bem sucedido ao mostrar que os materiais disponíveis para escolha dos professores do Ensino Médio estão cada vez mais incorporando conhecimentos de Física Moderna e Contemporânea (FMC). Como critério de seleção, está previsto que as coleções apresentem tópicos de FMC "que sejam considerados importantes ou mesmo imprescindíveis para o exercício da cidadania ativa, crítica e transformadora, bem como para a inserção ativa, crítica e transformadora no mundo do trabalho" (PNLD, 2015, p. 16).

Esse condicionante traz à tona elementos relevantes da Alfabetização Científica (AC). Sasseron e Carvalho (2011) enfatizam que, apesar de existirem na literatura diferentes teorizações sobre a AC, em síntese as habilidades que se deseja promover a partir dela podem ser organizadas em três eixos, quais sejam: (1) compreensão básica dos termos, conhecimentos e conceitos físicos fundamentais; (2) compreensão da natureza da ciência e dos fatores éticos e políticos que circundam sua prática, e; (3) entendimento das relações existentes entre ciência, tecnologia, sociedade e meio ambiente. As justificativas para inserção da FMC na Educação Básica são demasiadamente conhecidas, e muitas delas confluem para esses norteadores da AC.

Conforme sistematizado por Ostermann e Moreira (2000), há um caráter motivacional na introdução da Física Moderna e Contemporânea nas escolas, e também uma potencialidade para humanização dessa Ciência. Ainda, acrescentam os autores, através dela pode-se 
aproximar os estudantes do "excitante mundo da pesquisa atual em Física" e "atrair jovens para a carreira científica".

Complementando a ideia de humanização da Ciência, a inserção de tópicos da FMC na escola contribui ainda para que outros ideais da epistemologia contemporânea possam permear esse espaço educativo, tais como a não linearidade e o papel dos problemas na produção de conhecimentos científicos, a coletividade, a pluralidade metodológica, a divergência de pensamentos, etc.

Muitos dos textos originais de cientistas como Max Planck, Niels Bohr, trazem à tona momentos de conflito desses estudiosos diante do novo que despontava de suas pesquisas, situadas em um período histórico de transição entre duas formas bastante distintas de pensamento científico: a ciência clássica e a emergente física moderna. Materiais como esses podem contribuir grandiosamente para debates acerca dos valores na atividade científica, os quais podem ser subsidiados por uma variedade de correntes epistemológicas, tais como as de Kuhn (1989), Laudan (1984), Lacey (2008), Longino (1990) ou McMullin (1983).

O presente trabalho parte da preocupação com a mudança na visão de mundo que exige a física moderna, e com a ampla abstração necessária para a compreensão de seus conceitos. Sabe-se que a física clássica exige imaginação por parte dos estudantes, uma vez que pauta-se em idealização e lida com aspectos que não são observáveis diretamente, como campos elétricos, magnéticos, etc. Mas a constatação de um fenômeno e a compreensão de que a ciência clássica é limitada ao descrevê-lo, exigindo um novo sistema teórico, não soa como tarefa cognitiva trivial.

Diante disso, escreveu-se esse estudo pensando em professores da Educação Básica, e estudantes de graduação em Física. Apresenta-se para esse público uma breve contextualização histórica, que situa o surgimento do modelo atômico de Bohr. Na sequência, mostra-se que é possível utilizar esse modelo para descrever o estado fundamental e os estados excitados do átomo de hélio, mas evidencia-se nessa aplicação algumas limitações do modelo. Por fim, apresenta-se uma proposta de construção de um espectrômetro de baixo custo, que pode ser utilizado por professores em sala de aula, e problematiza-se alguns dados coletados a partir do experimento, bem como a conexão destes com a discussão do modelo atômico de Bohr à luz da mecânica quântica.

\section{Uma breve introdução histórica}

Desde as primeiras constatações newtonianas, a decomposição da luz tornou-se alvo de investigações científicas cada vez mais acuradas. Segundo Peduzzi (2015, p. 103), "em 1802 William H. Wollaston (1766-1828) observa a presença de linhas escuras no espectro solar, ao passar a luz por uma fenda estreita e depois por um prisma". Sucede a ele Joseph von Fraunhofer (1787-1826), que intensificou esses estudos, dando especial atenção às singelas linhas escuras que interrompiam o espectro da luz solar.

Outro marco na história da espectroscopia foram os trabalhos de Robert Bunsen 
(1811-1899) sobre as cores emitidas na queima de certos sais, o que despertou, de acordo com Peduzzi (2015, p. 103-104), o interesse de Gustav Kirchhoff (1824-1887). Os cientistas constataram que a cada elemento estava associado um espectro particular, e abriram os caminhos para a identificação da composição do Sol e de outras estrelas.

Na consolidação da espectroscopia destacam-se ainda o estudo do espectro solar e as medidas do espectro do hidrogênio feitas por Anders Jonas Angstrom (1814-1874), a equação empírica para determinação das linhas do hidrogênio formulada por Johann Jakob Balmer (1825-1898) a partir dos dados de Angstrom, e a equação de Johannes Robert Rydberg (18541919), que permitia predizer os comprimentos de onda emitidos pelo átomo de hidrogênio.

As constatações advindas da espectroscopia, os experimentos com raios catódicos, os desenvolvimentos no âmbito da radioatividade, e a descoberta do elétron por Joseph John Thomson (1856-1940) em 1987 clamavam por uma estrutura atômica mais complexa do que a proposta por John Dalton (1766-1844) em 1803. Assim, ao final do século XIX e início do século XX, emergiram diversas teorizações sobre essa possível estrutura, das quais foram protagonistas cientistas como o próprio Thomson, Hantaro Nagaoka (1865-1950), Ernest Rutherford (1871-1937), Niels Henrick David Bohr (1885-1962) dentre outros. É importante salientar que o desenvolvimento dos diversos modelos atômicos não se deu de forma linear, ou cumulativa. As datas em que foram propostos os distintos modelos mostram trabalhos quase que concomitantes e independentes. Apesar disso, nota-se a circulação das informações entre os cientistas pelas referências feitas em suas publicações.

Joseph J. Thomson propôs seu modelo atômico em 1904, no qual considerava que "os átomos dos elementos consistem de um número de corpúsculos eletrizados negativamente contidos em uma esfera de eletrificação uniforme e positiva" (THOMSON, 1904, p. 237, tradução nossa).

Nesse mesmo ano, Nagaoka propunha, em seu trabalho intitulado "Kinetics of a system of particles illustrating the Line and the Band Spectrum and the Phenomena of Radioactivity", um sistema atômico concebido sob influência do tratado de James Clerk Maxwell (1831-1879) sobre a estabilidade dos anéis de Saturno. Ele explica que o sistema

\begin{abstract}
consiste em um grande numero de partículas de mesma massa dispostas em um circulo, em intervalos angulares iguais, repelindo umas às outras por forças inversamente proporcionais ao quadrado da distância; no centro do círculo situa-se uma partícula de grande massa, atraindo as outras particulas de acordo com a mesma lei de força (NAGAOKA, 1904, p. 445, tradução nossa).
\end{abstract}

Ainda, o que difere o modelo de Nagaoka do sistema saturniano proposto por Maxwell é o fato de ele ter considerado partículas que se repelem, ao invés de satélites que se atraem. Peduzzi (2015, p. 175) explica que a intenção de Nagaoka era de interpretar "a) os espectros ópticos, atribuindo as linhas espectrais a oscilações desses anéis e b) a emissão radioativa de partículas por átomos pesados, vinculando o decaimento à quebra do anel exterior de elétrons do átomo pesado". 
Entre 1908 e 1913, Hans Geiger (1882-1945) e Ernest Marsden (1889-1970) realizavam, sob a supervisão de Rutherford em seu laboratório na Universidade de Manchester, uma série de experimentos que conduziram a um novo olhar sobre a estrutura atômica, como bem retratado por Parente, Santos e Tort (2013).

É em 1911 que Rutherford publica a memória em que descreve o seu modelo, segundo o qual, "os átomos são constituídos por um núcleo carregado positivamente, rodeado por um sistema de eléctrões ${ }^{3}$ ligados pelas forças atractivas do núcleo; a carga negativa total dos electrões é igual à carga positiva do núcleo" (BOHR, 2001, p. 95). Ainda, é o núcleo que concentra praticamente toda a massa atômica, mesmo possuindo dimensões muito pequenas em relação às dimensões totais do sistema.

Em proposições estritamente clássicas, como as de Rutherford e anteriores, deve-se levar em conta o teorema de Earnshaw, que prova não haver estabilidade atômica quando seus componentes - núcleo e elétrons - encontram-se estáticos. Rutherford concebe os elétrons em movimento orbital em torno do núcleo, e é nesse ponto que encontra-se uma contradição. Os elétrons em movimento acelerado emitem radiação o que implica na perda de energia pelo sistema atômico. O decréscimo na energia de ligação com o núcleo leva a um aumento na frequência de translação dos elétrons, que consequentemente migrariam de forma contínua para órbitas cada vez menores.

O processo continuará até que as dimensões da órbita sejam da mesma ordem de grandeza que as dimensões do eléctrão ou do múcleo. Um cálculo simples mostra que a energia irradiada durante o processo considerado será extremamente grande em comparação com a que é irradiada nos processos moleculares ordinários (BOHR, 2001, p. 99).

Niels Bohr estabelece uma aproximação com Rutherford quando vai trabalhar no laboratório em Manchester. Conforme biografia escrita por Cockcroft (1963), o cientista foi para lá após uma tentativa anterior de trabalho com J. J. Thomson, em Cambridge. "Durante as suas primeiras semanas no Laboratório ele assistiu a um curso organizado por Geiger, Makower e Marsden sobre métodos experimentais na pesquisa em radioatividade, para o benefício dos estudantes e visitantes" (p. 38, tradução nossa). Mas, como mostra o autor, Bohr logo se envolveu com problemas de natureza teórica que estavam sendo tratados no Laboratório.

Em 26 de julho de 1912, segundo a mesma fonte, Niels Bohr retornou a Copenhagen onde casou-se. Logo após o casamento, a caminho para a lua de mel na Escócia, parou em Manchester para entregar a Rutherford um manuscrito em que trabalhava. No retorno, o cientista dedicou-se novamente ao problema que mais lhe interessava: a questão da instabilidade do átomo de Rutherford perante a eletrodinâmica clássica. Cabe lembrar que modelos anteriores já vislumbravam dificuldade semelhante, mas é do modelo de Rutherford que Bohr parte, como explicado em seus escritos intitulados "On the constitution of atoms and molecules"

\footnotetext{
${ }^{3}$ Utilizou-se, neste estudo, uma tradução portuguesa dos trabalhos de Niels Bohr.
} 
(Sobre a constituição de átomos e moléculas), publicados em três partes na revista Philosophical Magazine de julho, setembro e novembro de 1913, respectivamente.

$\mathrm{Na}$ introdução da primeira parte de seu trabalho, Bohr fundamenta-se no modelo de Rutherford, explicando que irá admitir a estrutura do átomo com um núcleo concentrado, positivamente carregado e contendo parte significativa da massa desse átomo. Em seguida, apresenta o problema da instabilidade eletrodinâmica, o qual propõe resolver com o aporte da teoria de Max Planck (1858-1947) para, por fim, "(...) explicar de maneira simples a lei do espectro de riscas do hidrogênio" (BOHR, 2001, p. 97).

A argumentação de Bohr parte da concepção de um elétron suficientemente afastado de um determinado núcleo positivo, de modo que a energia de interação inicial e a frequência de revolução possa ser desconsiderada. Em determinado instante, esse elétron passa a orbitar estacionariamente o núcleo, transladando com frequência $\omega$. A partir da suposição de que "durante a ligação do eléctrão, é emitida uma radiação homogênea de frequência $v$, igual à metade da frequência de revolução do elétron na sua órbita final (...)" (Ibid., p 100), e considerando a equação de Planck $(E=h v)$, Bohr propõe que a energia $(W)$ que deve ser fornecida ao sistema para remover o elétron a uma grande distância do núcleo é dada por

$$
W=\tau h \frac{\omega}{2}
$$

sendo $\tau$ um número inteiro, $\mathrm{h}$ a constante de Planck e $\omega$ a frequência de revolução do elétron na órbita. É a partir daí, como mostram detalhadamente Parente, Santos e Tort (2013), que Bohr deduz teoricamente a equação empírica de Balmer para o espectro do hidrogênio, passando pela necessidade de quantizar o momento angular. Ele afirma que "somos levados a admitir que estas configurações corresponderão a estados do sistema nos quais não há radiação de energia; estados que consequentemente serão estacionários enquanto o sistema não for perturbado do exterior" (BOHR, 2001, p. 101).

Na segunda parte de seu trabalho, publicada em setembro de 1913, Bohr extrapola a discussão feita para o átomo de Hidrogênio a átomos de mais elétrons. Ele admite as partículas $\alpha$ como idênticas ao núcleo do átomo de hélio e passa a discutir como se dá por elas a captação de elétrons até a condição de neutralidade. Partindo da constatação de Rutherford de que a carga elétrica do núcleo corresponde, por átomo, a um número de elétrons aproximadamente igual a metade do peso atômico (Ibid., p. 134), Bohr postula que "os electrões estão dispostos em intervalos angulares iguais, rodando sobre anéis coaxiais em torno do núcleo" (Id., p. 134). Aplicando as suas considerações sobre o momento angular já aliadas à quantização estabelecida por Planck, ele presume as frequências de revolução dos elétrons e as dimensões das órbitas. Mesmo sem quase qualquer dado experimental que dê suporte à sua teoria para estes sistemas atômicos, Bohr continua desenvolvendo sua discussão sobre a estabilidade das órbitas eletrônicas e as energias de formação de átomos como o hélio.

Na sequência do presente estudo, apresenta-se os elementos fundamentais do modelo atômico de Bohr e as suas implicações na descrição do átomo de hélio, indo um pouco além 
do que fez o próprio Bohr nessa segunda parte de seu trabalho de $1913^{4}$.

\section{A estrutura do átomo de hélio}

Originalmente, Niels Bohr sintetiza os pressupostos de seu trabalho em dois postulados, quais sejam

1) Que o equilibrio dinâmico dos sistemas nos estados estacionários pode ser discutido por meio da mecânica ordinária, enquanto a passagem dos sistemas entre diferentes estados estacionários não pode ser tratada nessa base.

2) Que este último processo é seguido pela emissão de uma radiação homogénea, para a qual a relação entre a frequência e a quantidade de energia emitida é a dada pela teoria de Planck (Ibid., p. 104).

Valendo-se deles, desenvolve-se na sequência as equações de energia para os estados do átomo de Bohr. Admita-se, portanto, um átomo de um elétron, com carga nuclear $Z$, conforme representado na Fig. 1. Para ele, pode-se escrever a equação de equilíbrio mecânico:

$$
\frac{1}{4 \pi \varepsilon_{0}} \frac{Z e^{2}}{r^{2}}=\frac{m v^{2}}{r}
$$

que implica diretamente em

$$
v^{2}=\frac{Z e^{2}}{4 \pi \varepsilon_{0} m r}
$$

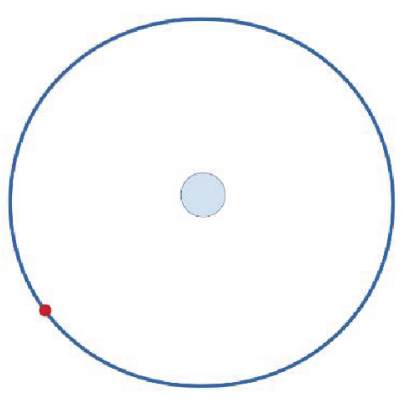

Fig. 1 -Representação esquemática de um átomo de um elétron, conforme o modelo de Bohr. Os tamanhos do elétron, núcleo e o diâmetro da órbita não estão em escala especifica.

As expressões (2) e (3) são estritamente clássicas, pois são construídas a partir da ideia de equilíbrio de forças. Porém, é possível avançar na descrição clássica do sistema nú-

\footnotetext{
${ }^{4}$ A terceira parte do trabalho de Niels Bohr, publicada na revista Philosophical Magazine em novembro de 2013 não será mencionada neste artigo, pois refere-se a estudos moleculares que eventualmente serão explorados em trabalhos futuros.
} 
cleo mais elétron, escrevendo as energias cinética e potencial como:

$$
\begin{gathered}
T=\frac{1}{2} m v^{2}=\frac{1}{2} \frac{Z e^{2}}{4 \pi \varepsilon_{0} r}, \\
V=-\int \vec{F} \cdot d \vec{r}=\int \frac{1}{4 \pi \varepsilon_{0}} \frac{Z e^{2}}{r^{2}} d r=-\frac{Z e^{2}}{4 \pi \varepsilon_{0} r} .
\end{gathered}
$$

Pode-se reconhecer que as expressões acima respeitam o Teorema do Virial, no caso particular em que a força entre as partículas do sistema é central e inversamente proporcional ao quadrado da distância entre elas. Na condição mais geral de força central $\left(F=C r^{n}\right)$, a relação entre a energia cinética e potencial é:

$$
T=\frac{1+n}{2} V
$$

que reduz-se ao caso presente na condição em que $n=-2$. Com isso, a energia total do sistema pode ser escrita como:

$$
E=-T=-\frac{1}{2} \frac{Z e^{2}}{4 \pi \varepsilon_{0} r}
$$

Mesmo não sendo a quantização do momento angular um resultado expresso explicitamente nos postulados originais de Bohr, essa ideia é fundamental para simplificar o argumento. A aplicação da quantização do momento angular, $L=n \hbar$, sobre a equação (3), implica diretamente na existência de órbitas bem definidas nas quais o elétron se move, em termos da sua velocidade e do raio orbital representado em função do número quântico n:

$$
\begin{gathered}
r=\frac{4 \pi \varepsilon_{0} \hbar^{2} n^{2}}{e^{2} Z m} \\
v=\frac{Z e^{2}}{4 \pi \varepsilon_{0} \hbar n}
\end{gathered}
$$

Utilizando essa expressão para o raio orbital (equação 8) na equação 7, encontra-se que

$$
E=-\frac{m Z^{2}}{2 \hbar^{2}}\left(\frac{e^{2}}{4 \pi \varepsilon_{0}}\right)^{2} \frac{1}{n^{2}} .
$$

De fato, ao empregar a ideia de que a energia é emitida pelo sistema atômico durante a transição do elétron entre diferentes estados representados pelo número quântico $n$, obtémse a expressão que descreve a frequência $(v)$ da radiação emitida no processo de transição entre os estados $n_{i}$ e $n_{f}$, tal que $n_{i}>n_{f}$.

$$
v=\frac{Z^{2} m}{4 \pi \hbar^{3}}\left(\frac{e^{2}}{4 \pi \varepsilon_{0}}\right)^{2}\left(\frac{1}{n_{f}^{2}}-\frac{1}{n_{i}^{2}}\right) .
$$


A expressão (11) representa muito bem o espectro de emissão de radiação visível (série de Balmer) e infra-vermelha (série de Paschen) pelo átomo de hidrogênio, quando se assume $Z=1$, resultado constatado e explicitado por Bohr na primeira parte de seu trabalho de 1913.

Ainda, essa equação fundamenta as séries espectrais observadas posteriormente, tais como as detectadas por Theodore Lyman (1874-1954) e August Herman Pfund (1879-1949), e permite verificar a origem da série de Pickering ao considerar $Z=2$, ou seja, ao descrever o espectro do átomo de hélio mono ionizado (He II).

Diante dessas deduções fica evidente que o modelo atômico proposto por Bohr é dotado de grande poder descritivo dos espectros de emissão de átomos de um elétron. Entretanto, alguns textos de física básica, especialmente aqueles voltados para o Ensino Superior, mencionam (sem argumentos muito claros) a inadequação desse modelo na descrição de sistemas simples de dois elétrons, como o átomo de hélio neutro. Considera-se que é preciso avançar e verificar, então, como ficam as expressões anteriores na descrição do átomo de hélio neutro (He I), e, consequentemente, averiguar a adequação da teoria de Bohr na descrição deste sistema.

A grande dificuldade na representação do átomo de hélio neutro a partir do modelo de Bohr reside na resolução do problema de três corpos que interagem por uma força central. Esta condição limita, inclusive, a determinação analítica da estrutura eletrônica do átomo de hélio (ou qualquer outro que tenha 2 ou mais elétrons), a partir da equação de Schrödinger.

$\mathrm{Na}$ configuração de menor energia, tem-se a restrição das coordenadas dos elétrons atômicos, os quais devem se mover solidariamente em uma trajetória circular (ou aproximadamente circular) em torno do núcleo, segundo o modelo de Bohr. Com essas condições iniciais, a estabilidade da trajetória é garantida. Para tal trajetória, o sistema físico pode ser representado como na Fig. 2.



Fig. 2 -Representação esquemática de um átomo de hélio, segundo o modelo atômico de Bohr. Novamente, os tamanhos dos elétrons, do núcleo e seus componentes, e o diâmetro da órbita não estão em escala especifica.

Cada um dos elétrons está individualmente sujeito as condições de equilíbrio, o que implica em: 


$$
-\frac{e^{2}}{4 \pi \varepsilon_{0}(2 r)^{2}}+\frac{Z e^{2}}{4 \pi \varepsilon_{0} r^{2}}=m \frac{v^{2}}{r}
$$

e, por consequência,

$$
v^{2}=\frac{e^{2}}{4 \pi \varepsilon_{0} m r}(Z-1 / 4)
$$

A energia cinética total, isto é, a energia cinética devido ao movimento dos dois elétrons é, portanto, dada por:

$$
T=\frac{1}{2} \sum_{i} m_{i} v_{i}=\frac{e^{2}}{4 \pi \varepsilon_{0} r}(Z-1 / 4)
$$

Para determinar a energia potencial a que os elétrons estão sujeitos pode-se utilizar novamente o Teorema do Virial. Entretanto, é mais ilustrativo demonstrar que este teorema é respeitado, pois:

$$
V=V_{l N}+V_{2 N}+V_{12}=-2 \frac{e^{2}}{4 \pi \varepsilon_{0} r}(Z-1 / 4),
$$

ou seja,

$$
T=-\frac{1}{2} V
$$

Como implicação, a energia total dos elétrons é:

$$
E=-T=-\frac{e^{2}}{4 \pi \varepsilon_{0} r}(Z-1 / 4) \text {. }
$$

Utilizando mais uma vez a regra de quantização do momento angular, obtém-se que:

$$
r=\frac{4 \pi \varepsilon_{0} \hbar^{2} n^{2}}{e^{2}(Z-1 / 4) m}
$$

e, consequentemente,

$$
E=-2(Z-1 / 4)^{2} \frac{m}{2 \hbar^{2}}\left(\frac{e^{2}}{4 \pi \varepsilon_{0}}\right)^{2} \frac{1}{n^{2}} .
$$

A essa altura é importante verificar que a energia do estado fundamental do átomo de hélio neutro dada pelo modelo de Bohr é 6,125 vezes maior que a energia do estado fundamental do átomo de hidrogênio. Ou seja, para o átomo de hélio $E_{B o h r}^{H e}=-83,3 \mathrm{eV}$.

Essa energia desvia apenas $5 \%$ do valor experimental atualmente aceito, qual seja $E_{e s p}^{H e}=-78,975 \mathrm{eV}$ (GRIFFITHS, 2011). O dado experimental para a energia do estado fundamental do hélio não existia em 1913, mas Bohr pôde verificar a precisão da equação acima comparando-a com o primeiro potencial de ionização do hélio, determinado por James Franck (1882-1964) e Gustav Hertz (1887-1975) mais cedo naquele mesmo ano (FRANCK; HERTZ, 
1913).

Uma vez que a energia de primeira ionização I é definida como a diferença entre as energias de formação $\mathrm{W}=-\mathrm{E}$ dos estados fundamentais dos átomos genéricos A I e A II,

$$
\mathrm{I}_{1}^{\mathrm{A}}=\mathrm{W}_{1}^{\mathrm{AI}}-\mathrm{W}_{1}^{\mathrm{AII}}=-\mathrm{E}_{1}^{\mathrm{AI}}+\mathrm{E}_{1}^{\mathrm{AII}}
$$

pode-se utilizar as equações representadas em (19) e (20) para demonstrar que, para um átomo A de carga nuclear $Z$,

$$
\mathrm{I}_{1}^{\mathrm{A}}=\left(2(\mathrm{Z}-1 / 4)^{2}-\mathrm{Z}^{2}\right) \mathrm{W}_{1}^{\mathrm{H}}
$$

Em particular, para um átomo de hélio $(Z=2)$, a energia de primeira ionização é igual a

$$
\mathrm{I}_{1}^{\mathrm{He}}=2,125 \mathrm{~W}_{1}^{\mathrm{H}}=28,9 \mathrm{eV}
$$

Este mesmo resultado, salvo ordens de aproximação numérica, foi demonstrado por Bohr na segunda parte de seu trabalho de 1913, onde verificou consistência na ordem de grandeza do valor obtido pela sua teoria com o valor determinado experimentalmente por Franck e Hertz no artigo previamente citado, qual seja

$$
\mathrm{I}_{\mathrm{He}}=20,5 \mathrm{eV} \text {. }
$$

Cabe ressaltar que este resultado experimental foi atualizado, e hoje é aceito como sendo aproximadamente igual a $24,587 \mathrm{eV}$ (ver por exemplo, KANDULA et al., 2010) ${ }^{5}$.

Além dos pontos discutidos acima, é importante verificar que o estado do átomo de hélio em que os dois elétrons se movem em uma órbita com $L=2 \hbar$ não é estável, pois a energia desse estado é maior que a energia de primeira ionização. Ou seja, o processo de ionização é favorecido em lugar da excitação simultânea dos dois elétrons do átomo de hélio.

Ao contrário do que acontece para o átomo de hidrogênio, a equação dos níveis de energia (19) para o átomo de hélio não pode ser utilizada para determinar o seu espectro de emissão, uma vez que esta expressão foi desenvolvida com a consideração de que distância entre os dois elétrons é sempre igual ao dobro da distância de um destes ao núcleo. Para avançar na análise do espectro do átomo de hélio, será considerada uma aproximação, no sentido de ilustrar, a partir desse momento, as limitações do modelo de Bohr.

Os níveis de energia de um átomo de dois elétrons podem ser descritos somando-se a energia de um átomo hidrogenóide no estado fundamental com a energia de um segundo elétron, em uma órbita de Bohr com $n>1$, de modo que, na média, o elétron mais externo pode ser considerado como sujeito à interação de uma carga nuclear efetiva $Z-1$, uma aproximação relativamente forte. Isto implica em

$$
E=-R h c Z^{2}-\frac{R h c(Z-1)^{2}}{n^{2}}
$$

\footnotetext{
${ }^{5}$ Outra fonte de referência pode ser a base NIST - Atomic Spectra Database Ionization Energies Form. Disponível em: <http://physics.nist.gov/PhysRefData/ASD/ionEnergy.html>. Acesso em: 20 out. 2016.
} 
onde, com o intuito de simplificar a notação, utilizamos a constante de Rydberg multiplicada pela constante de Planck e pela velocidade da luz

$$
R h c=\frac{m}{2 \hbar^{2}}\left(\frac{e^{2}}{4 \pi_{0}}\right)^{2} .
$$

Mesmo que a aproximação proposta acima seja forte e produza diferenças importantes nas energias de transições para valores pequenos de $n$, ela torna-se cada vez melhor conforme $n$ cresce. $\mathrm{O}$ espectro obtido a partir destes níveis de energia é tal que

$$
\frac{1}{\lambda}=R(Z-1)^{2}\left(\frac{1}{n_{f}^{2}}-\frac{1}{n_{i}^{2}}\right) .
$$

Para verificar a adequação da expressão acima com o espectro visível de emissão do hélio, pode-se calcular os comprimentos de onda para $n_{f}=2$ e $n_{i}=3,4,5$ e 6 , obtendo, respectivamente, os valores $656,1 \mathrm{~nm}, 486,0 \mathrm{~nm}, 433,9 \mathrm{~nm}$ e 410,1 nm, que são os comprimentos de onda da série de Balmer do hidrogênio, como deveríamos esperar pela aproximação utilizada neste cálculo. Ao avaliar o espectro de emissão medido nesta faixa de comprimentos onda, percebeu-se que são observadas pelo menos 10 linhas. Essa comparação pode ser feita a partir de dados disponíveis na base NIST, já mencionada, ou também, por meio da atividade experimental descrita adiante.

Fica claro que as linhas espectrais obtidas pela expressão acima, além de concordarem marginalmente com o resultado experimental, são apenas uma fração daquelas observadas. Esse ponto demonstra, portanto, uma falha séria no poder descritivo do modelo atômico de Bohr: a incapacidade de descrição quantitativa dos espectros de emissão para átomos com mais de um elétron. Ainda que o modelo possua grande mérito na descrição das energias de estados fundamentais e energias de ionização para átomos com poucos elétrons, a referida falha surge de forma natural, pois a mecânica clássica, utilizada fortemente no modelo de Bohr, limita a descrição dos estados estacionários dos átomos.

De qualquer forma, pode-se inferir que a ideia de órbitas em que o momento angular deve ser quantizado representa muito bem a origem do espectro de emissão e absorção, restando demonstrar apenas a concordância com dados empíricos. Somado a isso, destaca-se a proximidade com que o modelo de Bohr descreve as energias de estado fundamental do hélio e também de alguns outros átomos (PARENTE; SANTOS; TORT, 2013)

\section{Proposta experimental para análise espectral do He}

As reflexões feitas até o presente momento objetivaram fornecer subsídios teóricos a professores e estudantes de graduação em Física acerca do contexto histórico no qual Niels Bohr propôs seu modelo atômico, em 1913, e das limitações desse modelo ao tratar de átomos de múltiplos elétrons, sugerindo a necessidade de uma nova interpretação física. 
Nesse tópico, pretende-se mostrar uma possível atividade experimental para ser realizada em sala de aula, com o apoio de materiais simples. Porém, cabe ressaltar que a transposição didática das informações aqui presentes para o contexto da Educação Básica dependerá do professor e da estratégia educativa que este vier a elaborar. Defende-se que há nessa discussão potencial para inserção da física moderna no ensino também em nível superior, uma vez que parte-se de estudos experimentais simples sobre os espectros atômicos, até um mais complexo tratamento teórico da questão.

Com o intuito de analisar o espectro de emissão do átomo de hélio (ou outros similares), incentiva-se construir um espectrômetro utilizando uma câmera fotográfica digital - ou uma câmera de aparelho celular - e uma rede de difração, que pode ser obtida a partir de um CD.

Conforme indicado na Fig. 3, estima-se conseguir efeito semelhante ao do equipamento mostrado na imagem (a). Para isso é preciso posicionar sobre a câmera (e) um pedaço da parte acrílica do $C D$, com a superfície metálica já removida (b,c e d). Para fixar a rede de difração sobre a lente da câmera, pode-se utilizar a própria capa protetora do aparelho celular (f), ou então fitas adesivas. As fotografias que compõe a Fig. 3 demonstram a montagem experimental realizada para a obtenção dos dados deste trabalho.

A resolução das linhas espectrais obtidas por meio desse aparato pode ser melhorada com a utilização de uma fenda colimadora ${ }^{6}$. Mas, para a avaliação do espectro do hélio, as linhas mais fracas não seriam visíveis. Mesmo sem a fenda, porém, o espectrômetro proposto anteriormente permite uma razoável determinação das linhas espectrais do hélio.

Com o espectrômetro montado, basta abrir o aplicativo controlador da câmera digital do celular e fotografar uma fonte de luz, que, no caso desse estudo, foi uma lâmpada espectral de hélio ligada a uma fonte de alta tensão. Essa lâmpada de hélio é o objeto mais difícil de adquirir para a realização do experimento, mas os laboratórios de física das universidades geralmente possuem este tipo de material. Outra alternativa é a aquisição da lâmpada a partir de sites de empresas especializadas em comercializar equipamentos de laboratórios de física. No presente estudo, o experimento foi realizado no Laboratório de Física Moderna da universidade de origem dos pesquisadores.

A fotografia do espectro (Fig. 4) permite a visualização direta de pelo menos 8 linhas espectrais na faixa do visível, o que é suficiente para indicar qualitativamente a inadequação do modelo de Bohr para a descrição do espectro do átomo de hélio. Para uma análise quantitativa deve-se utilizar algum software que possibilite a determinação direta das intensidades dos picos observados na fotografia (Fig. 4), e seus respectivos comprimentos de onda. Para este fim, utilizamos o software livre SciDAVis.

\footnotetext{
${ }^{6}$ Como consta no endereço <https://publiclab.org/wiki/spectrometer >. Acesso em: 30 out. 2016.
} 


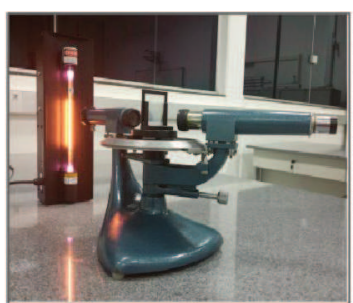

(a)

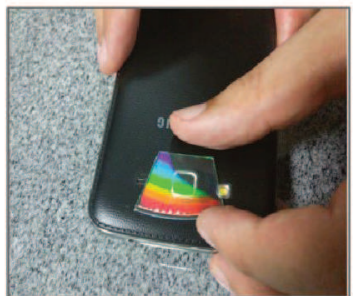

(e)

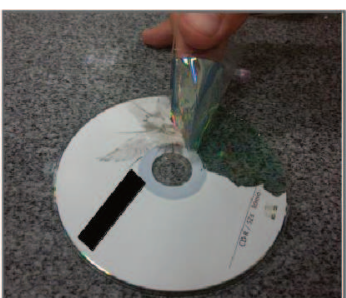

(b)

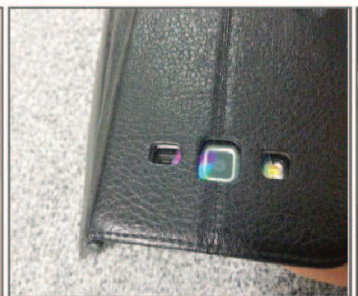

(f)

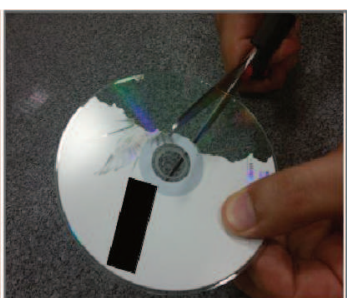

(c)

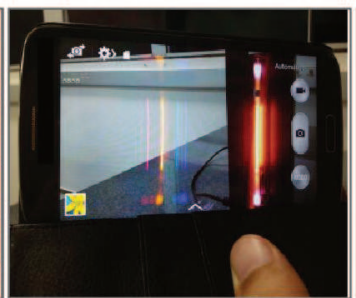

(g)

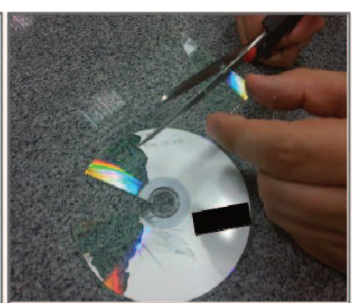

(d)

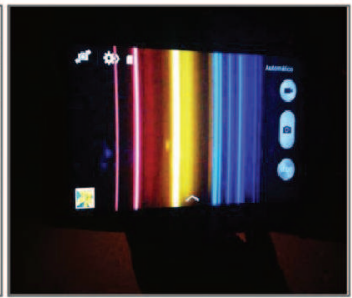

(h)

Fig. 3 -Montagem experimental e materiais utilizados. Em (a) aparece o aparelho que se deseja reproduzir com o CD e a câmera do celular. Em (b), (c) e (d) mostra-se a remoção da película espelhada do CD e o corte. Em (e) e (f) mostra-se como o CD pode ser fixado à câmera do celular. Em (g) e (h) vê-se o espectro aparecendo na tela do aparelho.

Para realizar a análise via SciDAVis, o primeiro passo consiste em importar a imagem no formato de matriz intensidade por pixel. Na opção matriz, seleciona-se conversão para tabela. Depois disso, marca-se todas as colunas da tabela, e, na aba análise, clica-se sobre estatística das colunas. Uma nova tabela surgirá, na qual a primeira coluna representa o número das colunas de pixels da fotografia, e a terceira coluna indica a média de intensidade para respectiva coluna de pixels.

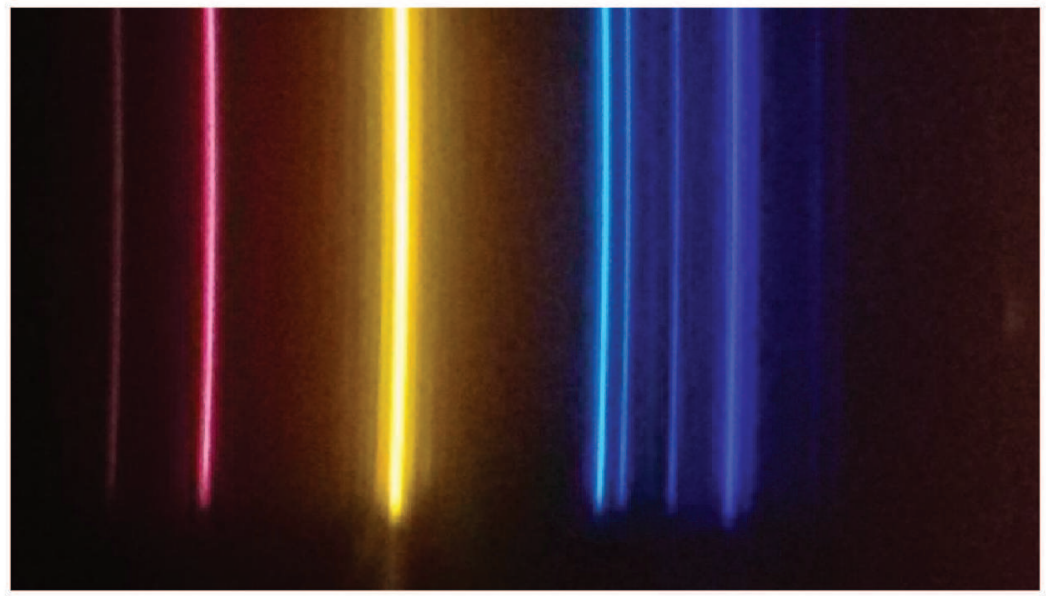

Fig. 4 - Fotografia do espectro do hélio, obtida conforme descrição da Fig. $3 .$. 
Fazendo o gráfico de intensidade média contra coluna de pixels, obtém-se o espectro apresentado na Fig. 5, em que é possível identificar facilmente 10 picos. A observação das linhas mais próximas do ultravioleta e do infravermelho, bem como as intensidades dos picos, dependem da sensibilidade da câmera utilizada e do pré processamento feito por cada aparelho.

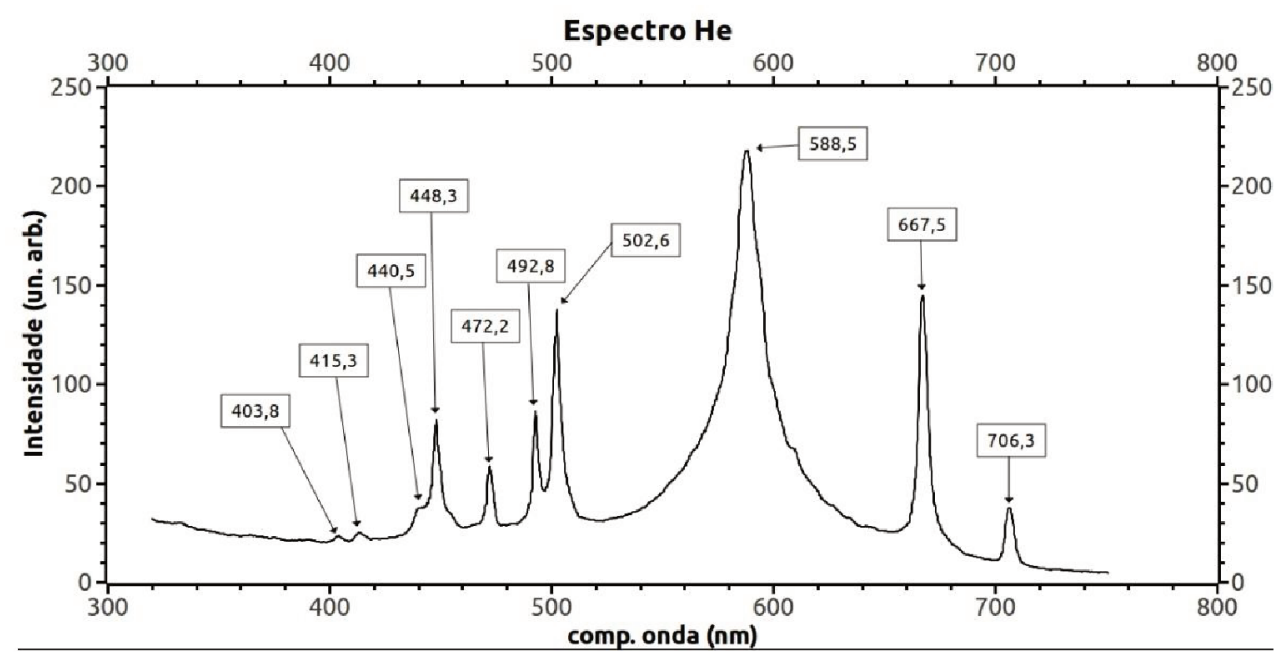

Fig. 5 - Curva de intensidades, já calibrada, obtida a partir da fotografia apresentada na Fig. 5.

Como o espectrômetro de celular aqui proposto não possui uma orientação fixa em relação à fonte de luz, deve-se calibrar cada fotografia individualmente para determinar o comprimento de onda dos picos encontrados. Para este fim, neste estudo utilizou-se o goniômetro também disponível no referido laboratório de Física Moderna. A partir dele, determinou-se os comprimentos de onda da linhas amarela $(588,5 \mathrm{~nm})$ e vermelha $(667,5 \mathrm{~nm})$ emitidas por esta mesma fonte.

Utilizando então os valores obtidos com o goniômetro como referência, e aplicando uma transformação linear nos valores das ordenadas (de pixel para comprimento de onda), foi possível determinar os comprimentos de onda das linhas espectrais observadas pelo aparato do celular, e compará-los com os valores aceitos.

Ao analisar os dados obtidos a partir do modelo atômico de Bohr, conforme delineado nas seções anteriores, percebeu-se que só é possível descrever razoavelmente 3 das raias espectrais medidas, quando se considera que o estado final do elétron excitado é $n_{\mathrm{f}}=2$ (série de Balmer para o He), assumindo uma carga nuclear efetiva $Z-a>1$ (assumir $Z-a<1$ é absurdo, pois significaria que, na média, o elétron interno possui carga maior que $e$ ). 
Tabela 1 - Valores medidos para as linhas de emissão do hélio em comparação com os valores experimentais aceitos. As linhas medidas $588,5 \mathrm{~nm}$ e $667,5 \mathrm{~nm}$, cujos valores aceitos são $587,6 \mathrm{~nm}$ e $667,8 \mathrm{~nm}$ respectivamente, não foram incluídas nesta tabela.

\begin{tabular}{|l|l|l|l|l|l|l|l|l|}
\hline Valor medido $(\mathrm{nm})$ & 403,8 & 415,3 & 440,5 & 448,3 & 472,2 & 492,8 & 502,6 & 706,3 \\
\hline Valor aceito $(\mathrm{nm})$ & 402,6 & 414,4 & 438,8 & 447,1 & 471,3 & 492,2 & 501,6 & 706,5 \\
\hline
\end{tabular}

Dessa forma demonstra-se claramente o ponto onde o modelo atômico de Bohr falha na descrição de sistemas atômicos com mais de um elétron: a justificação quantitativa do conjunto de raias espectrais em número de linhas e em comprimentos de onda. Ainda assim, o modelo atômico de Bohr possui grande mérito na determinação da energia do estado fundamental destes sistemas, não podendo ser considerado um total fracasso conceitual, conforme deixam a entender alguns livros texto voltados para disciplinas de física moderna dos cursos superiores em Física.

A comparação e discussão das raias espectrais dos átomos de hidrogênio e hélio produz uma excelente ponte para a introdução da teoria quântica ondulatória e equação de Schrödinger, além de explicitar a necessidade de introduzir, na descrição das funções de onda de sistemas atômicos, o spin dos elétrons e o princípio da exclusão de Pauli. Para exemplificar uma das questões que poderia ser discutida a partir dessas medidas de espectro, considere-se as diferenças de energia entre as transições medidas em relação às conhecidas transições da série de Balmer para o átomo de hidrogênio, como proposto por Abramzon e Siegel (2009), a partir da qual pode-se obter a seguinte tabela:

Tabela 2 - Diferença entre as energias das transições medidas a partir do experimento proposto em relação aos níveis conhecidos do átomo de hidrogênio. Valores comuns ao longo das três colunas estão destacados.

\begin{tabular}{|c|c|c|c|}
\hline$\lambda \mathrm{He}(\mathrm{nm})$ & $\Delta \mathrm{E}=\lambda \mathrm{He}-\mathrm{EH} \alpha(\mathrm{eV})$ & $\Delta \mathrm{E}=\lambda \mathrm{He}-\mathrm{H} \beta(\mathrm{eV})$ & $\Delta \mathrm{E}=\lambda \mathrm{He}-\mathrm{H} \gamma(\mathrm{eV})$ \\
\hline 403,8 & 1,18 & 0,52 & $\mathbf{0 , 2 2}$ \\
\hline 415,3 & 1,10 & 0,43 & 0,14 \\
\hline 440,5 & 0,93 & 0,26 & $\mathbf{- 0 , 0 3}$ \\
\hline 448,3 & 0,88 & $\mathbf{0 , 2 2}$ & $-0,08$ \\
\hline 472,2 & 0,74 & 0,08 & $-0,22$ \\
\hline
\end{tabular}

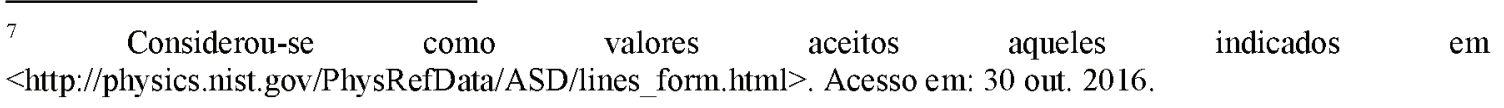




\begin{tabular}{|l|l|l|l|}
\hline 492,8 & 0,63 & $\mathbf{- 0 , 0 3}$ & $-0,33$ \\
\hline 502,6 & 0,57 & $-0,08$ & $-0,38$ \\
\hline 588,5 & $\mathbf{0 , 2 2}$ & -0.44 & $-0,74$ \\
\hline 667,5 & $\mathbf{- 0 , 0 3}$ & $-0,69$ & $-0,99$ \\
\hline 706,3 & $-0,13$ & $-0,80$ & $-1,09$ \\
\hline
\end{tabular}

É fácil observar na tabela 2 um conjunto de transições eletrônicas no átomo de hélio para as quais as diferenças, em termos de energia, em relação às transições eletrônicas no átomo de hidrogênio são constantes. De fato, se o espectrômetro utilizado pudesse registrar comprimentos de onda no ultravioleta, seria possível notar estas constâncias nas diferenças de energia também quando comparadas com as linhas mais energéticas do átomo de hidrogênio. O significado desta constância é que os estados finais do elétron nestas transições visualizadas para o átomo de hélio são fixos e próximos $(0,22 \mathrm{ou}-0,03 \mathrm{eV})$ do primeiro estado excitado do átomo de hidrogênio.

A partir das ideias introduzidas no contexto mecânica quântica - spin e princípio de exclusão - pode-se assumir que estes estados são correspondentes ao estado excitado para um elétron do átomo de hélio com $n=2$ (para ou orto-hélio), ou seja, correspondentes ao primeiro estado excitado do átomo de hidrogênio. A diferença de energia entre os níveis $n=2$ para $o$ átomo de hélio surge a partir dos diferentes estados de spin dos elétrons desta configuração. Escrevendo efetivamente as funções de onda para esses elétrons e utilizando da teoria de perturbação em primeira ordem, observa-se que a energia de um elétron em um estado excitado do átomo de hélio pode ser representada por (desconsiderando efeitos de blindagem):

$$
E=Z^{2} E_{100}+Z^{2} E_{n l m}+\Delta E,
$$

na qual $E_{n l m}$ é a energia de um átomo de hidrogênio cujos números quânticos são $n, l \mathrm{e} m, \mathrm{e}$

$$
A E=D \pm T
$$

é a soma ou a diferença das chamadas integrais direta $(D)$ e de troca $(T)$.

A avaliação destas integrais a partir de uma função de onda que descreva bem o sistema, permite demonstrar a diferença de energia entre os níveis $\mathrm{n}=2$ do átomo de hélio e $\mathrm{n}=2$ do átomo de hidrogênio.

Mesmo que o nível da descrição do átomo de hélio a partir da mecânica quântica seja complexo, as observações obtidas a partir de um experimento simples permitem demonstrar a necessidade desta teoria - em lugar do modelo atômico de Bohr - na descrição de um sistema atômico como o do hélio.

O desenvolvimento rigoroso da descrição deste sistema atômico a partir da mecânica quântica, ainda partindo destas simples observações experimentais realizáveis com um mínimo de materiais, será tema de um trabalho futuro. 


\section{Conclusões}

Quando se trata da inserção da física moderna na Educação Básica, alguns autores mostram-se contrários a enfatizar o modelo atômico de Bohr, pois entendem, em síntese, que "parte da dificuldade para a introdução dos conceitos quânticos deriva do fato de que, além de ser apresentada tardiamente, tende-se a enfatizar as diferenças e os contrastes com a Física Clássica, acontecendo, muitas vezes, que o aluno ignore os conceitos clássicos correspondentes" (GRECA; MOREIRA, 2001).

Na presente proposta, mostrou-se um breve delineamento histórico para retratar o cenário em que surgem os primeiros trabalhos de Niels Bohr sobre o modelo atômico. Os resultados da espectroscopia, sedenta por fundamentação teórica, mobilizaram diversos cientistas na tentativa de entender a estrutura atômica da matéria. Esse momento histórico por si só, apoiado por distintos referenciais da epistemologia contemporânea pode alavancar incessantes debates em sala de aula sobre a natureza da ciência ou então, como já mencionado, sobre os valores associados ao desenvolvimento científico. Essas discussões, com níveis de aprofundamento distintos, são pertinentes tanto para aulas de física na Educação Básica quanto no Ensino Superior, permitindo enfatizar a necessidade de uma nova física para explicar fenômenos conhecidos, ao invés de criar barreiras para a sua compreensão.

Ao falar de teorias do passado, muitos livros didáticos tendem a mostrá-las como facilmente descartáveis, ignorando a complexidade do contexto de descoberta, e, muitas vezes, refletindo um desconhecimento de seu poder explicativo e de predição. Evidenciou-se que o modelo de Bohr tem muito mais mérito na descrição dos sistemas atômicos do que apenas o ajuste do espectro dos átomos de um elétron. Ainda, demonstrou-se experimentalmente em que consiste a fragilidade da teoria de Bohr, ou seja, os efeitos de origem estritamente quântica que são diretamente conflitantes com o seu primeiro postulado.

Propôs-se um experimento simples a partir do qual podem emergir os fenômenos explicados pela mecânica quântica, cuja discussão criteriosa leva necessariamente ao domínio de novos conceitos ou aprofundamento das ideias vigentes. Não se espera que o professor da escola básica leve a profundidade e complexidade dessa discussão para a sala de aula, mas que ele encontre aqui fundamentação teórica - aliada a uma possibilidade experimental e a uma introdução histórica - para pautar variadas transposições didáticas.

\section{Referências}

ABRAMZON, N.; SIEGEL, P. B. Introductory helium atomic spectrum analysis. American Journal of Physics, v. 77, n. 10, 2009

BOHR, N. Sobre a constituição de átomos e moléculas. Tradução: Egídio Namorado. 4. ed. 
Lisboa: Fundação Calouste Gulbenkian, 2001.

COCKCROFT, J. D. Niels Henrik David Bohr. 1885-1962. Biographical Memoirs of Fellows of the Royal Society, p. 36-53, 1963.

EISBERG, R.; RESNICK, R. Física Quântica. 9. ed. Editora Campus, 1994. 928 p.

FRANCK, J.; HERTZ, G. Messung der ionisierungsspannung in verschiedenen Gasen. Verh. Phys. Ges. Berlin, n. 15, p. 4-44, 1913.

FREE SOFTWARE FOUNDATION. ScidAVis. 1991. Disponível em $<\mathrm{http}$ //scidavis.sourceforge.net/index.html>. Acesso em: 14 fev. 2016.

GRIFFITHS, D. Mecânica Quântica. 2. ed. Pearson Education, 2011.

GRECA, I. M.; MOREIRA, M. A. Uma revisão da literatura sobre estudos relativos ao ensino da Mecânica Quântica introdutória. Investigações em Ensino de Ciências, v. 6, n. 1, p. 29$56,2001$.

GUIA DE LIVROS DIDÁTICOS: PNLD 2015: física: ensino médio. - Brasília: Ministério da Educação, Secretaria de Educação Básica, 2014. 108p. Disponível em: $<\mathrm{http}$ ://www.fnde.gov.br/arquivos/category/125-guias?download=9003:pnld-2015-fisica $>$. Acesso em: 05 jan. 2016.

KANDULA, D. Z. et al. Extreme Ultraviolet Frequency Comb Metrology. Physical Review Letters, n. 105, 2010.

KUHN, T. A tensão essencial. Lisboa: Edições 70, 1989.

LACEY, H. Valores e atividade científica 1. 2. ed. São Paulo: Associação Filosófica Scientiae Studia/ Editora 34, 2008. 296 p.

LAUDAN, L. Science and values: the aims of science and their role in scientific debate. Berkeley, Los Angeles: University of California Press, 1984.

LONGINO, H. Science as a social knowledge: values and objectivity in scientific inquiry. Princeton: Princeton University Press, 1990.

McMULLIN, E. Values in Science. In: PSA: Proceedings of the Biennial Meeting of the Philosophy of Science Association. The University of Chicago Press, v. 2: Symposia and Invited Papers, 1983.

NAGAOKA, H. Kinetics of a system of particles illustrating the Line and the Band Spectrum and the Phenomena of Radioactivity. 1904. Disponivel em: $<$ http://www.plasma.uaic.ro/topala/articole/Nagaoka\%201904.pdf> Acesso em: 11 fev. 2016. 
OSTERMANN, F.; MOREIRA, M. A. Uma revisão bibliográfica sobre a área de pesquisa "física moderna e contemporânea no ensino médio". Investigações em Ensino de Ciências, v. 5, n. 1, p. 23-48, 2000.

PARENTE, F. A. G; SANTOS, A. C. F.; TORT, A. C. Os cem anos do átomo de Bohr. Revista Brasileira de Ensino de Física, v. 35, n. 4, 2013.

PEDUZZI, L. O. Q. Do átomo grego ao átomo de Bohr. 2015, 214 p. Disponível em: $<$ http://media.wix.com/ugd/7d71af_9315ee05281547c3ad821085a212467c.pdf $>$. Acesso em: 01 mar. 2016.

SASSERON, L. H.; CARVALHO, A. M. P. Alfabetização científica: uma revisão bibliográfica. Investigações em Ensino de Ciências, v. 16, n. 1, p. 59-77, 2011.

THOMSON, J. J. On the Structure of the Atom: an investigation of the Stability and Periods of Oscillation of a number of Corpuscles arranged at equal intervals around the Circumference of a Circle; with Applications of the results to the Theory of Atomic Structure. Philosophical Magazine, v. 7, n. 39, 1904. 